\title{
Preoperative central macular thickness as a risk factor for pseudophakic macular edema
}

\author{
C. J. Doncel-Fernández ${ }^{1}$ D • G. Castro-Luna ${ }^{2}$
}

Received: 4 November 2020 / Revised: 4 November 2020 / Accepted: 16 November 2020 / Published online: 26 November 2020

(C) Springer-Verlag GmbH Germany, part of Springer Nature 2020

First of all, we would like to thank Kanclerz, P et al. for their interest in reading our article and sharing their conclusions. We are pleased that he conducted a post hoc analysis of his previously published data in his study conducted on 95 eyes of 93 patients with diabetes undergoing routine cataract surgery at the Department of Ophthalmology, Kymenlaakso Central Hospital, Kotka, Finland.

By way of clarification, we would like to communicate that our study aimed to determine whether CMT before cataract surgery may be a risk factor itself for developing CME defined by the presence of intraretinal cysts after uncomplicated cataract surgery in patients with no previously known risk factors. The exclusion criteria were the presence of diabetic macular edema, thrombosis, or agerelated macular degeneration. The diabetic patients included in the study did not present diabetic retinopathy and had good metabolic control. Of the 179 patients who completed the study, $16.67 \%$ were diabetic. The level of $\mathrm{HbA1c}$ was $6.6( \pm 1.23)($ mean $\pm \mathrm{SD})$ with good levels of glycemia in the preoperative analysis $(99.8(16.7) \mathrm{mg} / \mathrm{dl})$ (mean $\pm \mathrm{SD}$ ). In our article no statistical significant differences were found in central macular thickness between diabetics and non-diabetics throughout the postoperative follow-up.

In our cohort of 179 eyes with no known risk factors, patients with pre-surgical macular thickness $>260.5 \mu \mathrm{m}$, measured by Cirrus Optical Coherence Tomography (OCT; Carl Zeiss Meditec AG, Jena, Germany), were 9-fold more likely to develop intraretinal macular cysts postoperatively. In the 95-eye cohort of Kanclerz, P et al., preoperative CMT was $287.9 \pm 55.6 \mu \mathrm{m}$ (mean $\pm \mathrm{SD}$ ) and that every micrometer of preoperative CMT correlated with a CMT increase of $0.089 \mu \mathrm{m}$ measured by spectral-domain OCT (Spectralis, Heidelberg Engineering GmbH, Heidelberg, Germany).

Both data confirm that preoperative central macular thickness is a risk factor for pseudophakic macular edema, and that the performance of OCT before cataract surgery may be justified in all patients.

Publisher's note Springer Nature remains neutral with regard to jurisdictional claims in published maps and institutional affiliations.

C. J. Doncel-Fernández

carlosjesus.doncel@ephpo.es

1 Department of Ophtalmology, Hospital Universitario de Poniente, Almeria, Spain

2 Department of Nursery, Physiotherapy and Medicine, University of Almería, Almeria, Spain 